
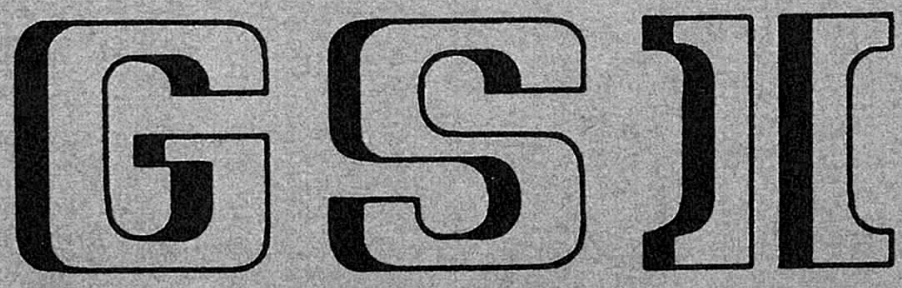

GSI-93-32 REPORT AUGUST 1993 ISSN 0171-4546

SOME REMARKS ON THE ION OPTICS OF BEAM DELIVERY SYSTEMS FOR TUMOR TREATMENT

J. JANIK, M. MÜLLER

Gesellschaft für Schwerionenforschung mbH Postfach 110552 - D-64220 Darmstadt · Germany 



\title{
Some Remarks on the Ion Optics of Beam Delivery Systems for Tumor Treatment
}

\author{
J.Janik \\ The Faculty of Electrical Engineering, \\ Slovak Technical University, Bratislava, Slovak Republic \\ M. Müller \\ Gesellschaft für Schwerionenforschung, Darmstadt, Germany
}

\begin{abstract}
The ion optics of a "Gantry-Illuminator" is presented and by an analytical formalism the guidelines and limits for the optimalisation of the system's main parameters are shown.
\end{abstract}

\section{Introduction}

Considering the layout of dedicated medical accelerators for the the tumor therapy with relativistic light ion beams [1], [2] it becomes evident that one the main subjects of interest is the beam delivery system to the patient. According to a highly specified irradiation plan [3] this beam delivery system transports the ion beam from the accelerator into the tumor creating the desired dose distribution. One of the demands coming from the irradiation plan is a narrow beam, as used in most practical cases. Another demand is the variability of the incident beam angle, which can be achieved by a rotatable gantry construction [4], [5], [6].

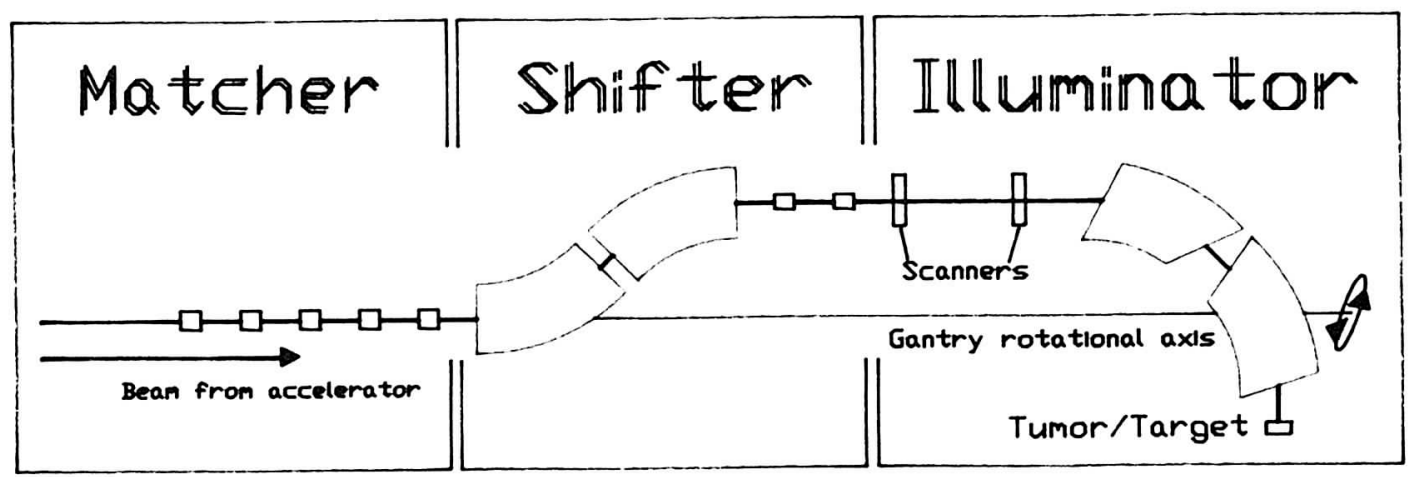

Figure 1: Schematic of Gantry Beam Delivery System 
Such a gantry, see Fig.1, may consist of three major groups, called MATCHER, SHIFTER and ILLUMINATOR [7]; the MATCHER, a series of magnetic quadrupole lenses to match the beam emittance to the Gantry acceptance, the SHIFTER, an antidirectional pair of magnetic dipoles to shift the beam parallel to the original direction and the ILLUMINATOR, to bend the beam finally to the target, producing a suitable beam shape in the tumor range. An incorporated "raster scan" device [3] sweeps the beam in $\mathrm{x}$ and $\mathrm{y}$ direction and creates a preset dose distribution. Together with the energy variability of the ion beam conformal radiation treatment of tumors can be achieved.

\section{The Ion Optics of the Illuminator.}

The illuminator may consists at least of one dipole (bending magnet) or of a system of dipole magnets, quadrupole magnets etc. Magnetic sweepers, to move the beam in the irradiation area, are placed to its focal points $\mathrm{F}_{1 x}$ and $\mathrm{F}_{1 y}$ to achieve a parallel shifting beam axis a.t the target [7]

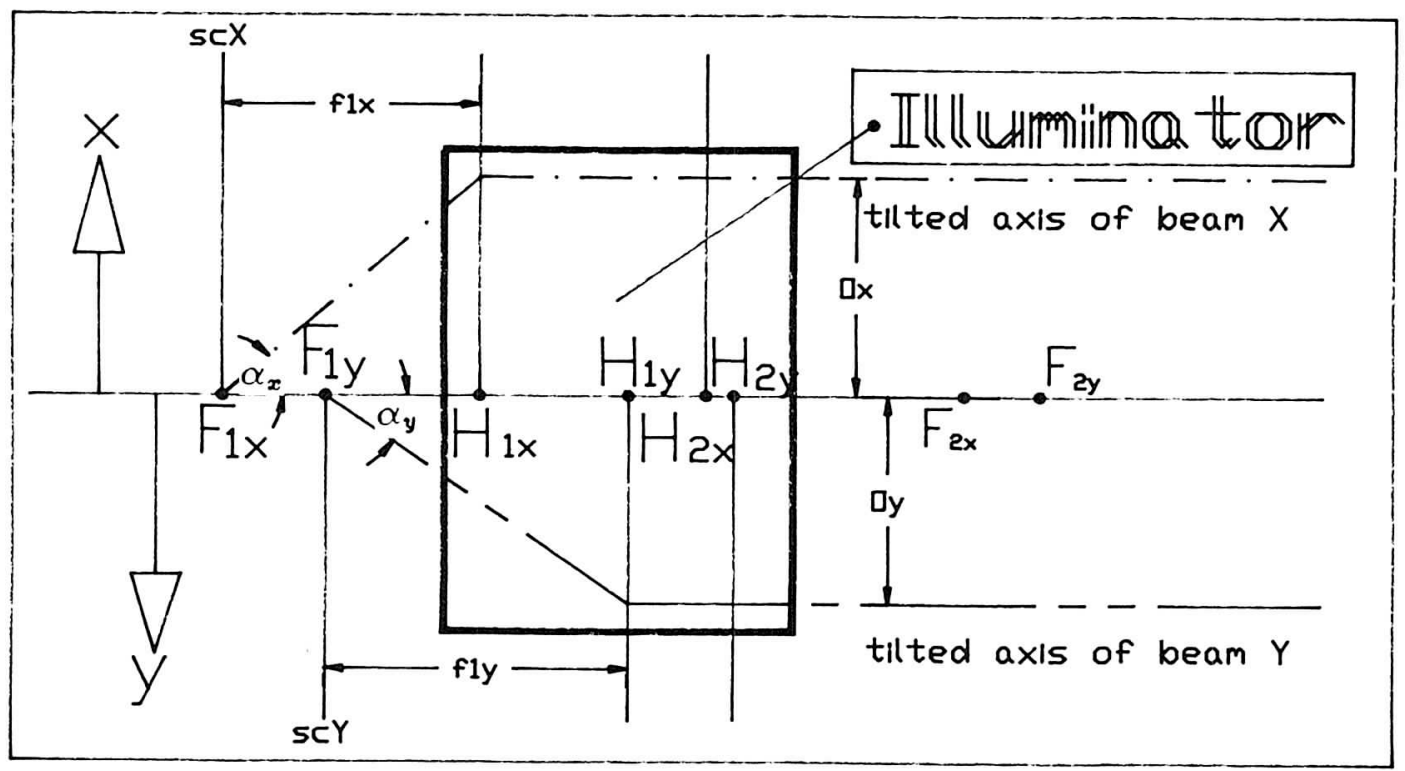

Figure 2: Schematic Graph of Illuminator Optics

The displacements $\mathrm{O}_{x}, \mathrm{O}_{y}$ of beam axis, due to sweeper action are given by the equations:

$$
\begin{aligned}
& O_{x}=f_{x} \times \tan \alpha_{x} \\
& O_{y}=f_{y} \times \tan \alpha_{y}
\end{aligned}
$$

where $\mathrm{f}_{x}, \mathrm{f}_{y}$ are focal distances (see Fig.2) $\alpha_{x}$ and $\alpha_{y}$ are the beam angles after sweeper passage. Usually the treatment demands for a small diameter, low divergent beam and we now are looking for quantitative limits of this beam for a given optical system and also for the optimum in positioning the tumor in the system. The illuminator [7] is ionopticaly described 
by the transformation matrix $M$, which is in general of dimension $(6 \times 6)$ [8] For simplification optical properties are seen only for plane xz. For the y-direction, plane yz, the situation is similar. Also dispersive properties of the bending Magnets are neglected (the dispersion function and its first derivative is set to zero by suitable settings of the lenses preceding the sweepers). Now $\mathbf{M}$ is merely of dimension $(2 \times 2)$. Furthermore it is advantageous for the calculation when the references points [9] are both set to the focal points $F_{1}$ and $F_{2}$ (see Fig.3).

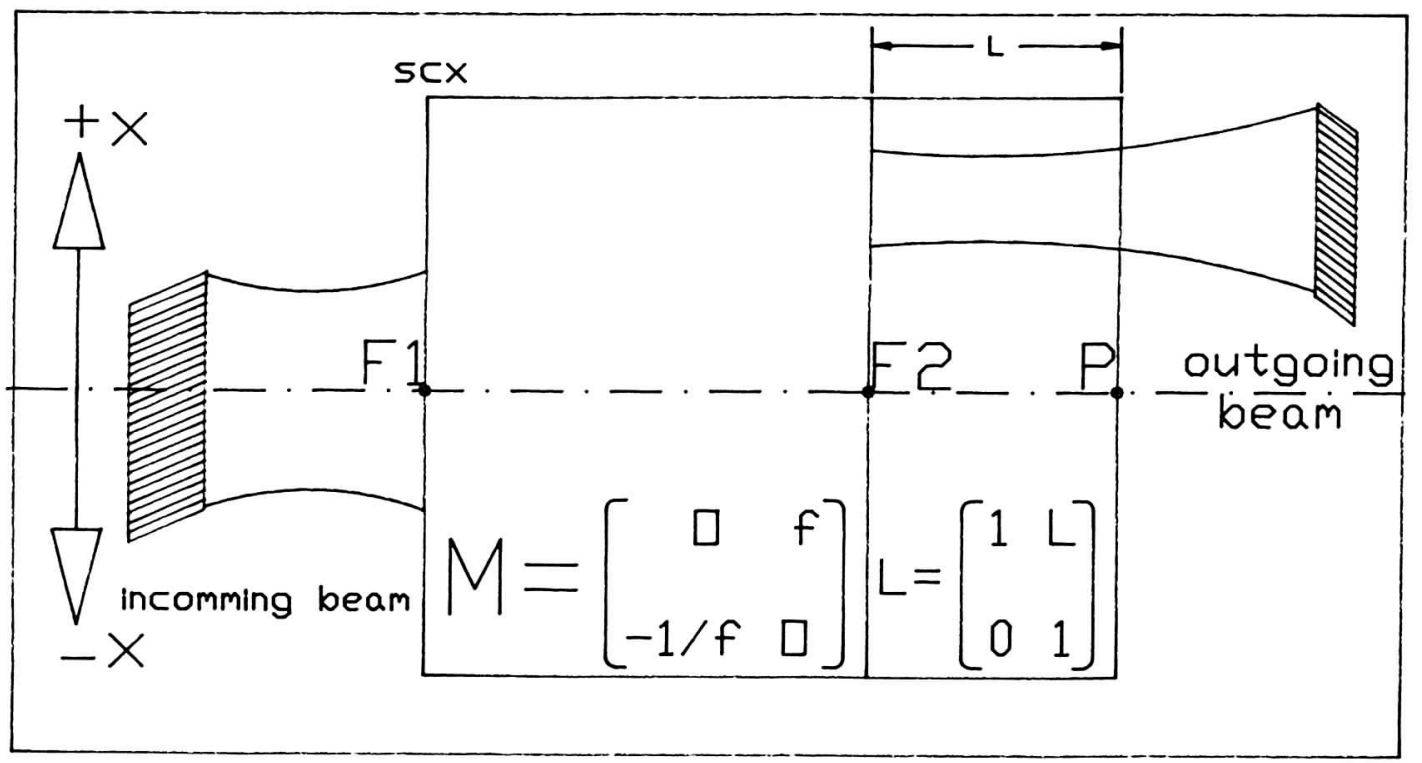

Figure 3: Matrix Schema for Illuminator Optics Calculations

Regarding the Illuminator as a lens, its transformation matrix $M$ from point $F_{1}$ to $F_{2}$ can be written as $[10]$.

$$
\mathbf{M}=\left(\begin{array}{cc}
0 & f_{1} \\
-1 / f_{2} & 0
\end{array}\right)
$$

$f_{1}$ and $f_{2}$ are its focal distances. In our case they are equal and therefore the indices " 1 " and "2" are omitted. The tumor is situated in the point $\mathrm{P}$ at distance $\mathrm{L}$ from focal point $\mathrm{F}_{2}$ (the value of $\mathrm{L}$ may be negative or zero too). The transformation matrix $\mathbf{R}$ from point $\mathrm{F}_{1}$ to point $\mathrm{P}$ is given by multiplication od the drift matrix $\mathbf{L}$ and the $\mathbf{M}$ matrix:

$$
\mathbf{R}=\left(\begin{array}{ll}
1 & L \\
0 & 1
\end{array}\right) \times\left(\begin{array}{cc}
0 & f_{1} \\
-1 / f_{2} & 0
\end{array}\right)
$$

If in the point $\mathbf{F}_{1}$ the beam is characterized by $\sigma(\mathrm{S})$ matrix

$$
\sigma(S)=\left(\begin{array}{ll}
\sigma_{11}(S) & \sigma_{12}(S) \\
\sigma_{21}(S) & \sigma_{22}(S)
\end{array}\right)
$$

then in the point $\mathrm{P}$ the matrix $\sigma(\mathrm{P})$ is given by

$$
\sigma(P)=\mathbf{R} \times \sigma(S) \times \mathbf{R}^{-T}
$$


Doing so, one gets

$$
\begin{gathered}
\sigma_{11}(P)=\frac{L^{2}}{f^{2}} \sigma_{11}(S)-2 L \sigma_{12}(S)+f^{2} \sigma_{22}(S) \\
\sigma_{12}(P)=\frac{L}{f^{2}} \sigma_{11}(S)-\sigma_{12}(S) \\
\sigma_{22}(P)=\frac{1}{f^{2}} \sigma_{11}(S)
\end{gathered}
$$

Lets now ask the question: Can the $\sigma_{11}(P)$, characterizing the size of the beam, reach zero value for a certain $L$ ? If the answer is yes the next equation must be fulfilled:

$$
\frac{L^{2}}{f^{2}} \sigma_{11}(S)-2 L \sigma_{12}(S)+f^{2} \sigma_{22}(S)=0
$$

This equation is quadratic in the variable $\mathrm{L}$, and if it is satisfied for some $\mathrm{L}$, then the discriminant $\mathrm{D}$ is not negative. Thus

$$
D=4 \sigma_{11}^{2}(S)-\left(\frac{2}{f}\right)^{2} \sigma_{11}(S) f^{2} \sigma_{22}(S)
$$

After simplifying and introducing the emittance by equ.

$$
\epsilon=\pi \times \varepsilon=\pi \times \sqrt{\operatorname{det} \sigma(S)}
$$

we get

$$
D=-4 \varepsilon^{2}
$$

For a realistic emittance $(\varepsilon>0)$, the discriminant is negative. This indicates that zero size of beam does not exist. The optimal distance for $\mathrm{L}$ is where $\sigma_{11}(\mathrm{P})$ is at minimum. It can be determined by setting the first derivative of $\sigma_{11}(\mathrm{P})$ to zero.

$$
\frac{d}{d L}\left(\sigma_{11}(P)\right)=0
$$

as follows from equ. (5)

$$
L=f^{2}\left(\frac{\sigma_{12}(S)}{\sigma_{11}(S)}\right)
$$

This place is the waist as we can almost see from equ.(6) setting $\sigma_{12}(\mathrm{P})=0$. Using equ. (11) in equ. (5) we get

$$
\sigma_{11}(P)=\frac{\varepsilon^{2} f^{2}}{\sigma_{11}(S)}
$$

After (12) the size of the output beam depends only on the ratio $f^{2}$ ans $\sigma_{11}(\mathrm{~S})$ because of emittance conservation. For a given $f$ one can always find a certain distance $L$ where the size $2 \mathrm{x}_{w}$ of the beam's waist is determined by 


$$
2 x_{w}=2 \sqrt{\frac{\varepsilon^{2} f^{2}}{\sigma_{11}(S)}}
$$

We can conclude now: The tumor has to be placed at a distance $\mathrm{L}$ from $\mathrm{F}_{2}$ given by equ.(11). Inside the tumor we can create a beam diameter of $2 \mathrm{x}_{w}$ given by equ.(13) with divergence given by equ.(7).

A surprising result has to be mentioned: At the place of the sweeper no waist is required, but when there is a waist in the sweeper, then $\mathrm{L}=0$. For given $\sigma(\mathrm{S})$ we can calculate the beam input parameters. For the treatment the beam parameters inside the tumor are important, described by the $\sigma(\mathrm{P})$ matrix. The task is now to find the $\sigma(\mathrm{S})$ matrix at the point where the beam enters the sweeper. For this purpose equ.(4) has to be multiplied from the left side by matrix $\mathbf{R}^{-1}$ (the inverse matrix of $\mathbf{R}$ ) and from the right side by $\mathbf{M}^{-R}$ (the inverse transposed matrix of $\mathbf{R}$ ).

$$
\sigma(S)=\mathbf{R}^{-1} \times \sigma(P) \times \mathbf{R}^{-T}
$$

The elements of matrix $\sigma(\mathrm{S})$ are:

$$
\begin{gathered}
\sigma_{11}(S)=f^{2} \sigma_{22}(P) \\
\sigma_{12}(S)=L \sigma_{22}(P)-\sigma_{12}(P) \\
\sigma_{22}(S)=\frac{L^{2}}{f^{2}} \sigma_{22}(P)-\frac{2 L}{f^{2}} \sigma_{12}(P)+\frac{1}{f^{2}} \sigma_{11}(P)
\end{gathered}
$$

From these equations one can easily determine the input beam requirements. A last remark: When $\sigma_{12}(\mathrm{~S})=0$ after equ.(1) $\mathrm{L}=0$ and after $(6)$ the $\sigma_{12}(\mathrm{P})=0$ too. For this case the transformation "waist-to-waist" occures and the well known relation is valid:

$$
X_{S} * X_{P}=f^{2}
$$

where $\mathrm{X}$ is the "characteristic length" of the beam, defined only in the waist by the equation:

$$
X_{w}=\sqrt{\frac{\sigma_{11}(w)}{\sigma_{22}(w)}}
$$

\section{Conclusion}

Necessary conditions for the design of a "Gantry-Illuminator" have been found. However for the realistic construction the use of a numerical computer code, TRANSPORT [8] e.g., is unavoidable but the amount of of time consuming work can be significantly reduced following the guidelines of the analytical results. 


\section{Status of the Gantry Design Activities}

Three main types of Gantries have been investigated: The Supraconducting Isocentrical Gantry, the Normalconducting Isocentric Gantry and the Normalconducting Excentric Gantry. All Gantries have been investigated with respect to integrated beam sweeping in both directions, small beam diameter and minimal divergence at the Gantry exit (tumor).

The supraconducting isocentric Gantry shows some advantages as were:

small over-all diameter: below 8 meters

small beam size

below $1 \mathrm{~mm}$ diameter

quadratic irradiation area $10 \mathrm{~cm} \times 10 \mathrm{~cm}$

and isocentric geometry.

The only disadvantage is the unfamiliar technology of Supraconductivity; many problems in this field remained unsolved up to now: The movement, due to mechanical rotation, of the superconducing Gantry may be critical (because of quenching). The manufacturing of big curved superconducting dipole magnets is not a standard technology. The fast field variation, unavoidable in the treatment operating, can be done with big effort only.

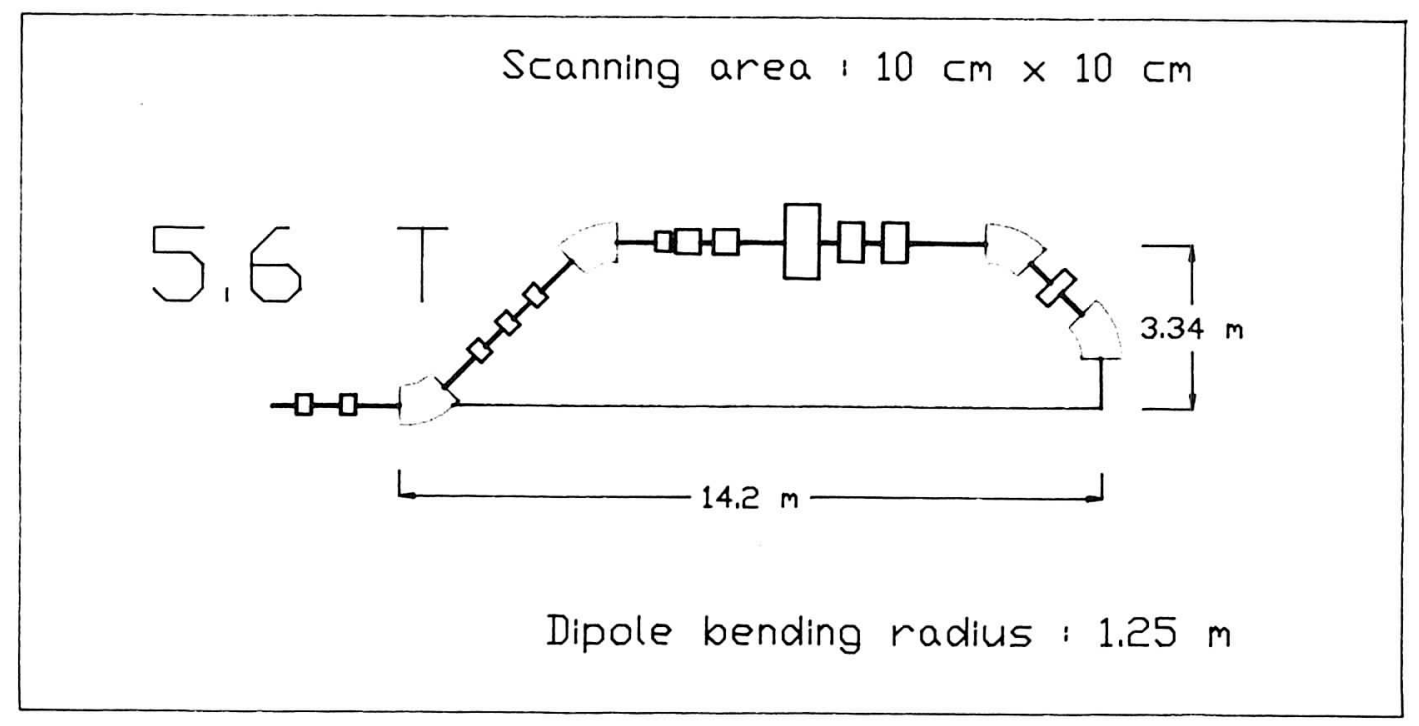

Figure 4: Schematic of Superconducting Isocentric Gantry

The normalconducting isocentric Gantry was mainly regarded as a model only, no not as serious alternative for realisation. The over-all diameter of at least $10 \mathrm{~m}$ and the weight of more then 80 tons give reason to look for other solutions, however the basic ion optical features had been clearly worked out with this layout. 


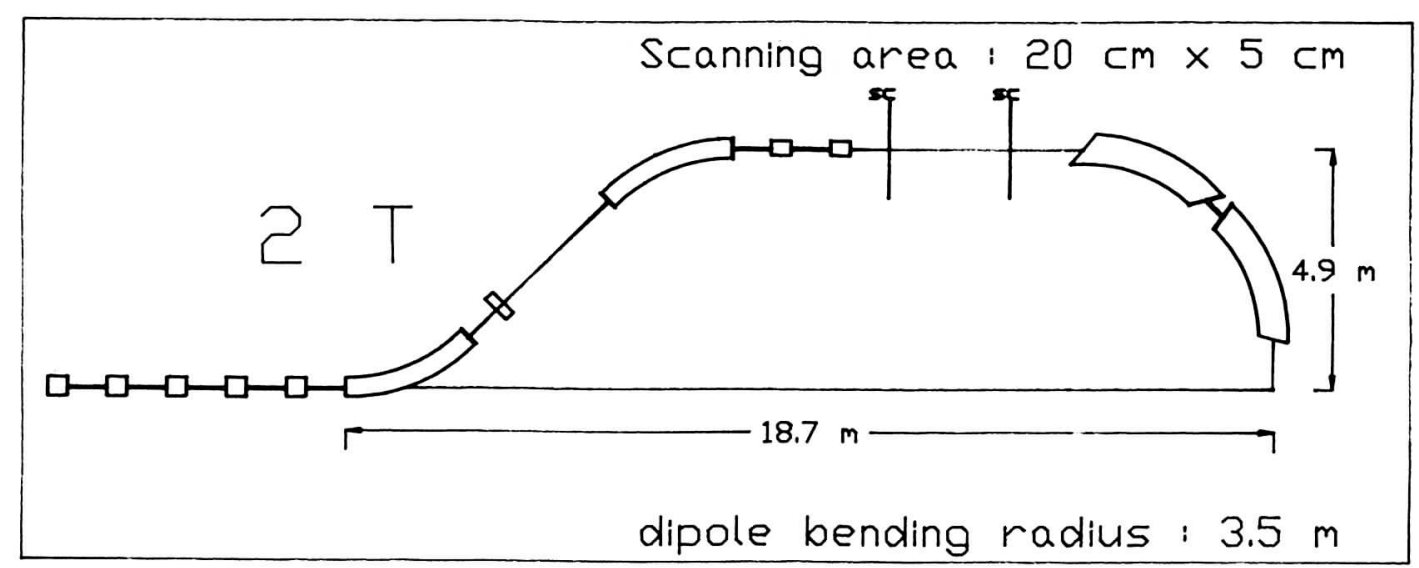

Figure 5: Normalconducting Isocentric Gantry

A way out of this dilema can be seen in the normalconducting excentric gantry already described by Pedroni [5] for Proton beams and by Carli [4] for Oxygen beams. This layout has the advantage of smaller over-all diameter but is inconvenient for the treatment itself, because the moving of the patient according to the selected gantry angle has to be accepted. Our construction is designed for $7 \mathrm{Tm}$ Neon beams. The irradiation area is $(5 \times 20) \mathrm{cm}^{2}$ and additionally this rectangular shape may be advantageous for many treatment conditions. Together with a traveling cabin, housing the treatment facilities, the over-all diameter of the gantry framework may stay below 7 meters. A slightly smaller solution using 2 Tesla dipole magnet field was also studied but stays frozen temporarily because of the unclear feasibility of such magnets.

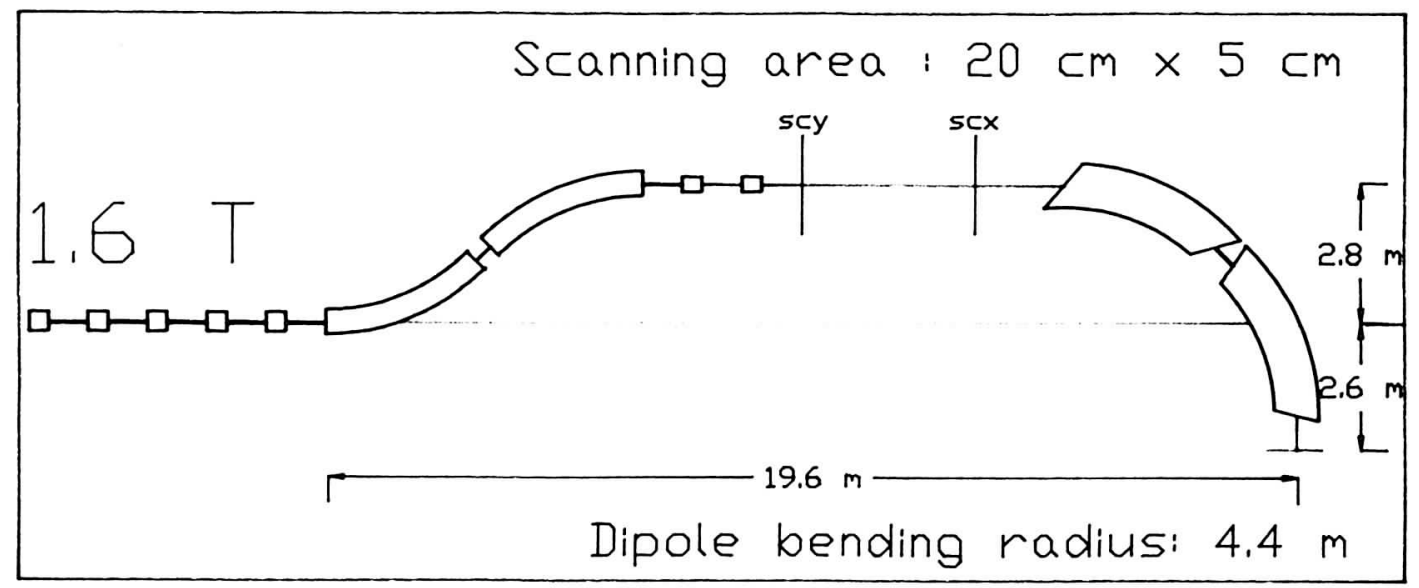

Figure 6: Normalconducting Excentric Gantry 


\section{Acknowledgements}

We are indebted to Dr. D. Böhne of GSI for his grateful support of this work and to Dr. B. Langenbeck of GSI for his useful advices and help.

\section{References}

[1] Böhne, D.: Light ion accelerators for cancer therapy, Radiat. Environ. Biophys (1992) $31205-218$

[2] MÜLLER, M.: Scientific Report 1991 GSI-Darmstadt

[3] BIOPHYSICS GROUP, GSI DARMSTADT: Design, Contribution and First Experiments of a Magnetic Scanning System for Therapy GSI-91-18, Report June 1991 ISSN $0171-4546$

[4] CARLI, Ch.: EULIMA, Preliminary Design of the Gantry for the EULIMA Project, Geneva 31.3.92

[5] PEDRONI, E., ENGE, H.: Beam Optics Design of a Compact Gantry for Proton Therapy, World Congress on Medical Physics and Biomedical Engineering, Kyoto, July 7-12, 1991

[6] MÜLLER, M., LANGENBECK,B.: Scientific Report 1992 GSI-Darmstadt p.337

[7] JANÍK, J.: Scientific Report 1992 GSI-Darmstadt, p.338

[8] BROWN, K. L. et al.: TRANSPORT CERN 73-16, Geneva 1973

[9] SEPTIER, A.: Focussing of charged particles. AP 1967

[10] LAWSON, J. D.: The Physics of Charged-Particle Beams, Clarendon Press Oxford 1988 